\title{
The Impacts of Population Subdivision on the Viability of Brachyteles hypoxanthus
}

\author{
Anderson A. Eduardo ${ }^{a}$ Daniel Brito ${ }^{b}$ \\ aprograma de Pós-graduação em Ecologia e Conservação da Biodiversidade, \\ Departamento de Ciências Biológicas, Universidade Estadual de Santa Cruz, Ilhéus, e

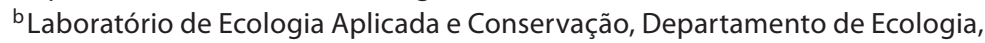 \\ Instituto de Ciências Biológicas, Universidade Federal de Goiás, Goiânia, Brasil
}

\author{
Key Words \\ Atlantic Forest • Habitat fragmentation • Habitat loss • Metapopulation dynamics • \\ Population viability analysis
}

\begin{abstract}
Habitat loss and fragmentation turn continuous large populations into metapopulations of smaller populations, more prone to the negative effects of stochastic processes. We modeled scenarios simulating the subdivision of Brachyteles hypoxanthus populations under different dispersal rates. Results show the existence of a population subdivision threshold, below which subdivision causes the metapopulation structure to collapse. Management should target first the increase in local populations through habitat restoration/protection, and only after populations are sufficiently large, connectivity strategies should take place.

Copyright $\odot 2012$ S. Karger AG, Basel
\end{abstract}

\section{Introduction}

Habitat fragmentation is one of the main threats to the world's biodiversity [Vié et al., 2009]. One of the consequences of habitat fragmentation is population subdivision, where one (or a few) large populations inhabiting a continuous habitat is converted into several small populations (or subpopulations) inhabiting the remnants of native habitat [Fahrig, 1997, 2001]. It is already known that small populations are more prone to suffer from stochastic events and processes [Lacy, 2000], and because of that may decline towards extinction [Hanski et al., 1996].

The Atlantic Forest is one of the world's ecosystems most impacted by habitat fragmentation [Myers et al., 2000; Mittermeier et al., 2005]. Estimates show that only between 11 and $16 \%$ of the original native vegetation cover is left [Ribeiro et al.,

\begin{tabular}{ll}
\hline KARGER & ( ) 2012 S. Karger AG, Basel \\
Fax +41 61 306 12 34 & \\
$\begin{array}{l}\text { E-Mail karger@karger.ch } \\
\text { www.karger.com }\end{array}$ & $\begin{array}{l}\text { Accessible online at: } \\
\text { www.karger.com/fpr }\end{array}$
\end{tabular}

Daniel Brito, Universidade Federal de Goiás Instituto de Ciências Biológicas Departamento de Ecologia Laboratório de Ecologia Aplicada e Conservação Goiânia, Goiás (Brasil), E-Mail brito.dan@gmail.com 
2009]. Brazil's major cities and economic centers, and most of the country's human population (approx. 70\% or 169 million), are found within the Atlantic Forest [Jacobsen, 2003]. As a result, what is left of the Atlantic Forest is scattered as small and isolated forest remnants [Ribeiro et al., 2009].

Large forest remnants within a fragmented landscape are important for preserving mammal community structure [Chiarello, 1999, 2000], but the role of small habitat remnants in maintaining native populations must also be taken into account when planning for species persistence [Turner and Corlett, 1996]. However, the effects of landscape alterations on population viability mediated by changes on population structure are highly complex and are still not fully understood.

The northern muriqui (Brachyteles hypoxanthus) is endemic to the Atlantic Forest [Mendes et al., 2005]. Northern muriqui populations were extirpated throughout the species range, mainly due to habitat loss, habitat fragmentation and hunting [Mendes et al., 2005]. Because of the severity of these impacts, nowadays only 13 highly fragmented and small populations persist [Mendes et al., 2005], and it is no surprise that the species is classified as 'Critically Endangered' in the IUCN's Red List of Threatened Species [IUCN, 2011]. Our objective is to model the impact of population subdivision, due to habitat fragmentation, on the viability of B. hypoxanthus.

\section{Methods}

Target Species

B. hypoxanthus is endemic to the Atlantic Forest [Aguirre, 1971]. It is highly folivorous but its diet also includes fruits, flowers and, to a lesser degree, other plant items such as bark [Strier, 1991a; Carvalho et al., 2004]. Muriquis are diurnal and arboreal, but they may descend to the ground to cross forest gaps, drink water and sometimes to play [Dib et al., 1997; Mourthé et al., 2007; Tabacow et al., 2009]. They are social and troops may have up to 100 individuals [Strier et al., 2006], with males being philopatric and females dispersing when they reach sexual maturity (6 years old) [Strier and Ziegler, 2000]. The mean age at first reproduction is estimated as 7 years for males and 9 years for females [Strier and Ziegler, 2000; Strier, 2005]. Females give birth to one baby and there is a 3-year interbirth interval [Strier, 1991b; Strier et al., 2001]. Longevity is estimated to be 35 years [Strier, 2000].

Population Viability Analysis Model and Scenarios

We used the software Vortex (version 9.92) [Lacy et al., 2008] to model our scenarios. Vortex is a Monte Carlo simulation of the effects of deterministic forces as well as demographic, environmental and genetic stochasticity and catastrophes on the dynamics of wildlife populations [Lacy, 1993; 2000; Lacy et al., 2008]. Our model incorporates stochasticity in mortality rates and reproductive rates (e.g. percentage of individuals reproducing in each year; for details on which parameters of the model are deterministic and which are stochastic, see Appendix). The use of population viability analysis models (PVA) to guide the management of the northern muriqui has been identified as one of the priority conservation actions for the species [Mendes et al., 2005].

Critical threshold population sizes for the persistence of $B$. hypoxanthus have already been identified [Brito and Grelle, 2006]. Population sizes of 40 and 700 individuals should ensure safety from the effects of demographic and genetic stochasticity, respectively [Brito and Grelle, 2006]. Given these data, the dynamics of populations of B. hypoxanthus with 40 and 700 individuals were explored. Dynamics of metapopulations (a group of connected populations of the same species found within a given region) where the original population size (40 and 700) was subdivided into 2, 4 and 8 populations, were modeled. The dispersal rates among populations were set as $0,0.05,0.10$ or 0.20 . In Vortex, dispersal rates specify the probability that a given 
Table 1. Results of the PVA for a $B$. hypoxanthus metapopulation size of 40 individuals for a time period of 50 generations, subdivided into 1,2, 4 or 8 subpopulations and connected by dispersal rates of $0,5,10$ or $20 \%$ per year

\begin{tabular}{lrllllc}
\hline $\begin{array}{l}\text { Sce- } \\
\text { narios }\end{array}$ & $\begin{array}{l}\text { Dispersal } \\
\text { rate, } \%\end{array}$ & $\begin{array}{l}\text { Population growth } \\
\text { rate } \pm \text { SD }\end{array}$ & $\begin{array}{l}\text { Probability } \\
\text { of extinction }\end{array}$ & $\begin{array}{l}\text { Population } \\
\text { size } \pm \text { SD, } n\end{array}$ & $\begin{array}{l}\text { Heterozygosity } \\
\pm \text { SD }\end{array}$ & $\begin{array}{l}\text { Time to extinc- } \\
\text { tion, generations }\end{array}$ \\
\hline $1 \times 40$ & - & $0.061 \pm 0.067$ & 0.000 & $39.33 \pm 2.01$ & $0.178 \pm 0.209$ & - \\
\hline $2 \times 20$ & 0 & $0.059 \pm 0.065$ & 0.042 & $31.74 \pm 10.95$ & $0.377 \pm 0.227$ & 36.24 \\
& 5 & $0.051 \pm 0.064$ & 0.008 & $34.39 \pm 8.02$ & $0.192 \pm 0.213$ & 35.18 \\
& 10 & $0.045 \pm 0.064$ & 0.006 & $35.47 \pm 6.78$ & $0.219 \pm 0.217$ & 37.16 \\
& 20 & $0.031 \pm 0.065$ & 0.032 & $35.14 \pm 7.65$ & $0.254 \pm 0.219$ & 39.38 \\
\hline $4 \times 10$ & 0 & $0.046 \pm 0.088$ & 0.980 & $0.20 \pm 1.36$ & $0.000 \pm 0.000$ & 19.71 \\
& 5 & $0.019 \pm 0.083$ & 0.954 & $0.59 \pm 2.94$ & $0.050 \pm 0.136$ & 24.11 \\
& 10 & $0.003 \pm 0.085$ & 1.000 & $0.00 \pm 0.00$ & $0.000 \pm 0.000$ & 12.10 \\
& 20 & $-0.018 \pm 0.093$ & 1.000 & $0.00 \pm 0.00$ & $0.000 \pm 0.000$ & 4.50 \\
\hline $8 \times 5$ & 0 & $0.017 \pm 0.096$ & 1.000 & $0.00 \pm 0.00$ & $0.000 \pm 0.000$ & 10.40 \\
& 5 & $-0.015 \pm 0.100$ & 1.000 & $0.00 \pm 0.00$ & $0.000 \pm 0.000$ & 5.36 \\
& 10 & $-0.026 \pm 0.101$ & 1.000 & $0.00 \pm 0.00$ & $0.000 \pm 0.000$ & 3.02 \\
& 20 & $-0.025 \pm 0.098$ & 1.000 & $0.00 \pm 0.00$ & $0.000 \pm 0.000$ & 2.15 \\
\hline
\end{tabular}

Scenarios indicate number of populations $\times$ population size.

individual will disperse from one population to another in a specific year [Lacy et al., 2008]. We completed 500 simulations for each scenario. A time frame of 50 generations was selected according to the biology of the target species [Armbruster et al., 1999]. Demographic parameters used as input to the model were based on previously published demographic data and PVA studies on B. hypoxanthus [Strier, 1991b, 1993/1994; Rylands et al., 1998; Strier, 2000, 2005; Brito and Grelle, 2006; Strier et al., 2006; Coutinho, 2007; Brito et al., 2008]. Here, a population is considered demographically viable if it presents $<10 \%$ extinction probability in 50 generations and genetically viable if it retains $>90 \%$ of its original genetic diversity in 50 generations [Foose et al., 1986; Soulé, 1987].

\section{Results}

No extinctions were observed when we modeled single continuous populations of 40 or 700 individuals (tables 1, 2). However, scenarios that included population subdivision resulted in a decrease in population growth rate and final metapopulation size, and an increase in population fluctuation and probability of extinction (tables 1,2). The results show that dispersal rates do not counter the negative effects of population subdivision in small populations (table 1). In particular, when subdivision was more severe, an increase in dispersal was not capable of negating deleterious effects from fragmentation (table 1).

Our results show that small populations (40 individuals) do not support subdivision (table 1). Even the smallest level of subdivision resulted in a decrease in time to extinction (table 1). The scenarios evaluating the subdivision of a large population

$\overline{78} \quad$ Folia Primatol 2012;83:76-84 $\quad$ Eduardo/Brito


Table 2. Results of the PVA for a B. hypoxanthus metapopulation size of 700 individuals for a time period of 50 generations, subdivided into 1,2, 4 or 8 subpopulations and connected by dispersal rates of $0,5,10$ or $20 \%$ per year

\begin{tabular}{lrlllll}
\hline $\begin{array}{l}\text { Sce- } \\
\text { narios }\end{array}$ & $\begin{array}{l}\text { Dispersal } \\
\text { rate, } \%\end{array}$ & $\begin{array}{l}\text { Population growth } \\
\text { rate } \pm \text { SD }\end{array}$ & $\begin{array}{l}\text { Probability } \\
\text { of extinction }\end{array}$ & $\begin{array}{l}\text { Population } \\
\text { size } \pm \text { SD, } n\end{array}$ & $\begin{array}{l}\text { Heterozygosity } \\
\pm \text { SD }\end{array}$ & $\begin{array}{l}\text { Time to extinc- } \\
\text { tion, generations }\end{array}$ \\
\hline $1 \times 700$ & - & $0.062 \pm 0.050$ & 0.000 & $698.63 \pm 7.99$ & $0.910 \pm 0.019$ & - \\
\hline $2 \times 350$ & 0 & $0.063 \pm 0.041$ & 0.000 & $696.39 \pm 9.52$ & $0.914 \pm 0.018$ & - \\
& 5 & $0.056 \pm 0.041$ & 0.000 & $696.88 \pm 9.27$ & $0.916 \pm 0.018$ & - \\
& 10 & $0.049 \pm 0.041$ & 0.000 & $696.27 \pm 9.84$ & $0.921 \pm 0.018$ & - \\
& 20 & $0.034 \pm 0.039$ & 0.000 & $691.04 \pm 13.41$ & $0.929 \pm 0.014$ & - \\
\hline $4 \times 175$ & 0 & $0.063 \pm 0.035$ & 0.000 & $696.45 \pm 9.22$ & $0.922 \pm 0.013$ & - \\
& 5 & $0.042 \pm 0.034$ & 0.000 & $690.43 \pm 11.52$ & $0.927 \pm 0.014$ & - \\
& 10 & $0.020 \pm 0.032$ & 0.000 & $679.21 \pm 17.87$ & $0.932 \pm 0.014$ & - \\
& 20 & $-0.023 \pm 0.063$ & 1.000 & $0.00 \pm 0.00$ & $0.000 \pm 0.000$ & 11.07 \\
\hline $8 \times 87$ & 0 & $0.063 \pm 0.032$ & 0.000 & $694.36 \pm 9.21$ & $0.934 \pm 0.008$ & - \\
& 5 & $0.013 \pm 0.029$ & 0.000 & $652.09 \pm 24.07$ & $0.932 \pm 0.014$ & - \\
& 10 & $-0.034 \pm 0.063$ & 1.000 & $0.00 \pm 0.00$ & $0.000 \pm 0.000$ & 7.36 \\
& 20 & $-0.050 \pm 0.067$ & 1.000 & $0.00 \pm 0.00$ & $0.000 \pm 0.000$ & 4.14 \\
\hline
\end{tabular}

Scenarios indicate number of populations $\times$ population size.

(700 individuals) shows that subdividing the original population into two subpopulations does not affect long-term persistence, regardless of the dispersal rates among them (table 2). Scenarios of highly subdivided populations with no dispersal or low dispersal rates do not have negative effects for the populations (table 2).

\section{Discussion}

Theoretical studies indicate that population subdivision negatively affects demographic and genetic parameters of wildlife populations [Shaffer, 1981]. PVA models based on empirical data also showed the same trend for some mammals [Lacy and Lindenmayer, 1995; Lindenmayer and Lacy, 1995a; Brito and Fonseca, 2007; Brito, 2009a]. In a metapopulation structure, the size of the constituent populations seems to be an important factor for long-term persistence [Fahrig, 1997, 1998, 2001; Hanski and Gaggiotti, 2004]. Our results clearly show that dispersal does not seem to play an important role in the persistence of subdivided muriqui populations.

The subdivision of small populations (40 individuals) was detrimental to their long-term persistence, depressing growth rate and increasing demographic stochasticity [Shaffer, 1981; Soulé, 1987; Brito, 2009b]. Enhancing connectivity through dispersal had the result of further increasing population instability through metapopulation stochasticity [Lindenmayer and Lacy, 1995a, b; Brito and Fonseca, 2007; Brito, 2009a]. Dispersal among populations may also generate synchronous population dynamics, where temporal fluctuations in population size coincide (e.g. all popula- 
tions decline or increase in size at the same time), and increased susceptibility to extinction [Heino et al., 1997; Bjørnstad et al., 1999]. Unfortunately we were not able to measure population synchrony in our model, but if it takes place our results underestimate the extinction risk due to dispersal-driven synchrony among populations.

From a demographic point of view, it is generally accepted that dispersal of individuals among populations has a positive effect on persistence [Brown and Kodrick-Brown, 1977; Simberloff et al., 1992; Frankham, 1995, 1999; Lande, 1998; Waite et al., 2005], even though the onset of other biological processes, such as disease transmission, might turn dispersal into a detrimental effect to population persistence. However, our results suggest that, even considering only demographic processes, there is evidence that a threshold exists below which stochastic events through metapopulation instability have a more powerful grip on population dynamics and dispersal might be detrimental to persistence [Lindenmayer and Lacy, 1995a, b; Lacy and Lindenmayer, 1995; Brito and Fonseca, 2007; Brito, 2009b]. In another PVA model, dispersal was not detrimental to the persistence of a small Alouatta palliata mexicana population (approx. 75 individuals) in a fragmented landscape with fragments ranging from 15 to 60 ha [Mandujano and Escobedo-Morales, 2008]. However, we observed detrimental effects for B. hypoxanthus, suggesting this process may also affect medium and large mammals.

Our results corroborate previous theoretical and empirical evidence that high dispersal rates may be detrimental to small populations in highly fragmented landscapes due to stochastic events [Lindenmayer and Lacy, 1995b]. In these cases, the loss of even a few individuals due to unsuccessful dispersal attempts may counteract the possible benefits of a metapopulation structure, increasing the likelihood of extinction [Lindenmayer and Lacy, 1995b]. In metapopulations comprising small populations, the mortality of dispersing individuals due to stochastic events taking place during dispersal represents a proportionally greater negative impact on population dynamics [Lindenmayer and Lacy, 1995b]. In such scenarios, each individual represents a greater proportion of the population, and as populations become smaller, the loss of individuals during dispersal (resulting in unsuccessful dispersal) will be more detrimental than possible rescue effects from successful dispersal attempts [Lindenmayer and Lacy, 1995b]. However, even though our theoretical modeling approach suggests the possible existence of such a metapopulation/connectivity threshold for northern muriquis, we must take into account that the dispersal rates used to model the populations are theoretical. We still understand very little about how muriquis move among fragments, and about how different matrix types translate into landscape permeability for the species, as well as having few estimates of dispersal rates and little information on dispersal behavior. Fieldwork focusing on muriqui dispersal capability (or lack of) is of paramount importance, not only for advancing knowledge about the biology of the species, but also for refining models on how the landscape structure might affect the species persistence, and if, as our model suggests, it might be susceptible to connectivity thresholds. Besides that, our results may be conservative, since we did not take into account several human activities such as hunting and fires that act synergistically with habitat fragmentation and are likely to affect northern muriqui populations in fragmented landscapes [Rylands et al., 1998].

These analyses warn that caution must be taken when devising conservation actions in highly fragmented landscapes, as to date commonplace strategies to counter 
fragmentation are based on improving connectivity through dispersal corridors. In the case of the northern muriqui, empirical data on dispersal are urgently needed. Before management strategies are put into place, it would be wise to evaluate the landscape context each remnant population inhabits in order to estimate if it is below or above the subdivision threshold. Then, we could identify the most adequate habitat management plan for each situation, focusing on habitat restoration [Pontual and Boubli, 2005] or habitat connectivity, according to the landscape configuration where the population occurs.

\section{Appendix}

B. hypoxanthus demographic data used as input values to Vortex. Extinction is defined as no animals of one or both sexes.

No inbreeding depression

First age of reproduction for females: 9, for males: 7

Maximum breeding age (senescence): 35

Sex ratio at birth (percent males): 35.600000

Polygynous mating

$100.00 \%$ of adult males in the breeding pool

$26.50 \%$ of adult females produce litters

$\mathrm{EV}$ in \% adult females breeding $=12.40 \mathrm{SD}$

Of those females producing litters, ...

$100.00 \%$ of females produce litters of size 1

$2.00 \%$ mortality of females between ages 0 and 1

$\mathrm{EV}$ in \% mortality $=1.000000 \mathrm{SD}$

$5.70 \%$ mortality of females between ages 1 and 2

$\mathrm{EV}$ in \% mortality $=2.800000 \mathrm{SD}$

$1.00 \%$ mortality of females between ages 2 and 3

$\mathrm{EV}$ in \% mortality $=1.000000 \mathrm{SD}$

$3.60 \%$ mortality of females between ages 3 and 4

$\mathrm{EV}$ in \% mortality $=1.800000 \mathrm{SD}$

$1.00 \%$ mortality of females between ages 4 and 5

$\mathrm{EV}$ in \% mortality $=1.000000 \mathrm{SD}$

$1.00 \%$ mortality of females between ages 5 and 6

$\mathrm{EV}$ in \% mortality $=1.000000 \mathrm{SD}$

$1.00 \%$ mortality of females between ages 6 and 7

$\mathrm{EV}$ in \% mortality $=1.000000 \mathrm{SD}$

$1.00 \%$ mortality of females between ages 7 and 8

$\mathrm{EV}$ in \% mortality $=1.000000 \mathrm{SD}$

$1.00 \%$ mortality of females between ages 8 and 9

$\mathrm{EV}$ in \% mortality $=1.000000 \mathrm{SD}$

$1.00 \%$ mortality of adult females $(9 \cdot$ age $\cdot 35)$

$\mathrm{EV}$ in \% mortality $=1.000000 \mathrm{SD}$

$4.80 \%$ mortality of males between ages 0 and 1

$\mathrm{EV}$ in \% mortality $=2.400000 \mathrm{SD}$

$5.60 \%$ mortality of males between ages 1 and 2

$\mathrm{EV}$ in \% mortality $=2.800000 \mathrm{SD}$

$11.80 \%$ mortality of males between ages 2 and 3

$\mathrm{EV}$ in \% mortality $=5.900000 \mathrm{SD}$ 
$1.00 \%$ mortality of males between ages 3 and 4

$\mathrm{EV}$ in $\%$ mortality $=1.000000 \mathrm{SD}$

$1.00 \%$ mortality of males between ages 4 and 5

$\mathrm{EV}$ in $\%$ mortality $=1.000000 \mathrm{SD}$

$1.00 \%$ mortality of males between ages 5 and 6

$\mathrm{EV}$ in $\%$ mortality $=1.000000 \mathrm{SD}$

$1.00 \%$ mortality of males between ages 6 and 7

$\mathrm{EV}$ in $\%$ mortality $=1.000000 \mathrm{SD}$

$1.52 \%$ mortality of adult males $(7 \cdot$ age $\cdot 35)$

$\mathrm{EV}$ in $\%$ mortality $=1.500000 \mathrm{SD}$

EVs may be adjusted to closest values possible for binomial distribution

$\mathrm{EV}$ in reproduction and mortality will be concordant

$\mathrm{EV}$ in carrying capacity $=0.00 \mathrm{SD}$

Deterministic population growth rate (based on females, with assumptions of no

limitation of mates, no density dependence, and no inbreeding depression):

$\mathrm{r}=0.063, \lambda=1.065, \mathrm{R} 0=3.402$

Generation time for females $=19.57$, for males $=17.87$

$\mathrm{EV}=$ Environmental variation; $\mathrm{SD}=$ standard deviation.

\section{Acknowledgments}

We thank Deborah M. Faria, Júlio E. Baumgarten and Leonardo C. Oliveira for their valuable comments and suggestions. Anderson A. Eduardo thanks the Fundação de Amparo à Pesquisa do Estado da Bahia (FAPESB) for the MSc scholarship. Daniel Brito's work is funded by CNPq (Project No. 305631/2009-8).

\section{References}

Aguirre AC (1971). O Mono Brachyteles arachnoides (E. Geoffroy). Rio de Janeiro, Academia Brasileira de Ciências.

Armbruster P, Fernando P, Lande R (1999). Time frames for population viability analysis of species with long generations: an example with Asian elephants. Animal Conservation 2: 69-73.

Bjørnstad ON, Ims RA, Lambin X (1999). Spatial population dynamics: analyzing patterns and processes of population synchrony. Trends in Ecology and Evolution 14: 427-432.

Brito D (2009a). Genetic consequences of population subdivision: the marsupial Micoureus paraguayanus (Mammalia: Didelphimorphia) as a case study. Zoologia 26: 684-693.

Brito D (2009b). Análise de viabilidade de populações: uma ferramenta para a conservação da biodiversidade no Brasil. Oecologia Brasiliensis 13: 452-469.

Brito D, Fonseca GAB (2007). Demographic consequences of population subdivision on the long-furred woolly mouse opossum (Micoureus paraguayanus) from the Atlantic Forest. Acta Oecologica 31: 60-68.

Brito D, Grelle CEV (2006). Estimating minimum area of suitable habitat and viable population size for the northern muriqui (Brachyteles hypoxanthus). Biodiversity and Conservation 15: 4197-4210.

Brito D, Grelle CEV, Boubli JP (2008). Is the Atlantic Forest protected area network efficient in maintaining viable populations of Brachyteles hypoxanthus? Biodiversity and Conservation 17: 32553268.

Brown JH, Kodrick-Brown A (1977). Turnover rates in insular biogeography: effect of immigration on extinction. Ecology 58: 445-449. 
Carvalho O, Ferrari S, Strier KB (2004). Diet of a muriqui group in continuous primary forest. Primates 45: 201-204.

Chiarello AG (1999). Effects of forest fragmentation of the Atlantic forest on mammal communities in south-eastern Brazil. Biological Conservation 89: 71-82.

- Chiarello AG (2000). Density and population size of mammals in remnants of Brazilian Atlantic forest. Conservation Biology 14: 1649-1657.

Coutinho BR (2007). Análise de viabilidade populacional do muriqui, Brachyteles hypoxanthus (Primates: Atelidae), em fragmentos de Mata Atlântica de Santa Maria do Jetibá, Espírito Santo. Vitória, Universidade Federal do Espírito Santo.

Dib LRT, Oliva AS, Strier KB (1997). Terrestrial travel in muriquis (Brachyteles arachinoides) across a forest clearing at Estação Ecológica de Caratinga, Minas Gerais, Brazil. Neotropical Primates 5: $8-9$.

Fahrig L (1997). Relative effects of habitat loss and fragmentation on population extinction. Journal of Wildlife Management 61: 603-610.

-Fahrig L (1998). When does fragmentation of breeding habitat affect population survival? Ecological Modelling 105: 273-292.

Fahrig L (2001). How much habitat is enough? Biological Conservation 100: 65-74.

Fahrig L (2002). Effect of habitat fragmentation on the extinction threshold: a synthesis. Ecological Applications 12: 346-353.

-Fahrig L (2003). Effects of habitat fragmentation on biodiversity. Annual Reviews of Ecology, Evolution and Systematics 34: 487-515.

Foose TJ, Lande R, Flesness NR, Rabb G, Read B (1986). Propagation plans. Zoo Biology 5: 139-146.

Frankham R (1995). Inbreeding and extinction: a threshold effect. Conservation Biology 9: 792-799.

-Frankham R (1999). Resolving conceptual issues in conservation genetics: the roles of laboratory species and meta-analyses. Hereditas 130: 195-201.

Hanski I, Moilanen A, Gyllenberg M (1996). Minimum viable metapopulation size. The American Naturalist 147: 527-541.

Hanski I, Gaggiotti OE (2004). Ecology, Genetics, and Evolution of Metapopulations. New York, Academic Press.

Heino M, Kaitala V, Ranta E, Lindström J (1997). Synchronous dynamics and rates of extinction in spatially structured populations. Proceedings of the Royal Society of London B 264: 481-486.

IUCN (2011). The IUCN Red List of Threatened Species. http://www.iucnredlist.org.

Jacobsen TR (2003). Populating the environment: human growth, density and migration in the Atlantic Forest. In The Atlantic Forest of South America: Biodiversity Status, Threats, and Outlook (Galindo-Leal C, Câmara IG, eds.), pp 426-435. Washington, Island Press.

Lacy RC (1993). Vortex: a computer simulation model for population viability analysis. Wildlife Research 20:45-65.

Lacy RC (2000). Considering threats to the viability of small populations using individual-based models. Ecological Bulletins 48: 39-51.

Lacy RC, Lindenmayer DB (1995). A simulation study of the impacts of population subdivision on the mountain brushtail possum Trichosurus caninus Ogilby (Phalangeridae: Marsupialia), in southeastern Australia. II. Loss of genetic variation within and between populations. Biological Conservation 73: 131-142.

Lacy RC, Borbat M, Pollak JP (2008). Vortex: A Stochastic Simulation of the Extinction Process. Brookfield, Chicago Zoological Society.

Lande R (1998). Anthropogenic, ecological and genetic factors in extinction and conservation. Researches on Population Ecology 40: 259-269.

Lindenmayer DB, Lacy RC (1995a). A simulation study of the impacts of population subdivision on the mountain brushtail possum Trichosurus caninus Ogilby (Phalangeridae: Marsupialia) in southeastern Australia. I. Demographic stability and population persistence. Biological Conservation 73: 119-129.

Lindenmayer DB, Lacy RC (1995b). Metapopulation viability of arboreal marsupials in fragmented oldgrowth forests: comparison among species. Ecological Applications 5: 183-199.

Lindenmayer DB, Burgman MA, Akçakaya HR, Lacy RC, Possingham HP (1995). A review of the generic computer programs ALEX, RAMAS/space and VORTEX for modelling the viability of wildlife metapopulations. Ecological Modelling 82: 161-174.

Lindenmayer DB, Lacy RC, Thomas VC, Clark TW (1993). Predictions of the impacts of changes in population size and environmental variability on Leadbeater's possum, Gymnobelideus leadbeateri McCoy (Marsupialia: Petauridae) using population viability analysis: an application of the computer program VORTEX. Wildlife Research 20: 67-86.

Mandujano S, Escobedo-Morales LA (2008). Population viability analysis of howler monkeys (Alouatta palliata mexicana) in a highly fragmented landscape in Los Tuxtlas, Mexico. Tropical Conservation Science 1: 43-62. 
Mendes SL, Melo FR, Boubli JP, Dias LG, Strier KB, Pinto LPS, Fagundes V, Cosenza B, De Marco P (2005). Directives for the conservation of the northern muriqui, Brachyteles hypoxanthus (Primates, Atelidae). Neotropical Primates 13 (suppl): 7-18.

Mittermeier RA, Gil PR, Hoffmann M, Pilgrim J, Brooks T, Mittermeier CG, Lamoreux J, Fonseca GAB (2005). Hotspots Revisited: Earth's Biologically Richest and Most Endangered Terrestrial Ecoregions. Mexico City, CEMEX.

Mourthé IMC, Guedes D, Fidelis J, Boubli JP, Mendes SL, Strier KB (2007). Ground use by northern muriquis (Brachyteles hypoxanthus). American Journal of Primatology 69: 706-712.

Myers N, Mittermeier RA, Mittermeier CG, da Fonseca GAB, Kent J (2000). Biodiversity hotspots for conservation priorities. Nature 403: 853-858.

- Pontual FB, Boubli JP (2005). The Caratinga Alliance: community-based conservation efforts to increase forest for the muriquis and water for the farmers. Neotropical Primates 13: 47-52.

Ribeiro MC, Metzeger JP, Martensen AC, Ponzoni FJ, Hirota MM (2009). The Brazilian Atlantic Forest: how much is left, and how is the remaining forest distributed? Implications for conservation. Biological Conservation 142: 1141-1153.

Rylands A, Strier K, Mittermeier R, Borovansky J, Seal US (1998). Conserving Brazil's Muriqui: Population and Habitat Viability Assessment Workshop for the Muriqui (Brachyteles arachnoides). Apple Valey, Conservation Breeding Specialist Group.

Shaffer ML (1981). Minimum population sizes for species conservation. Bioscience 31: 131-134.

- Simberloff DA, Farr JA, Cox J, Mehlman DW (1992). Movement corridors: conservation bargains or poor investments? Conservation Biology 6: 493-504.

Soulé ME (1987). Viable Populations for Conservation. Cambridge, Cambridge University Press.

- Strier KB (1991a). Diet in one group of wooly spider monkeys, or muriquis (Brachyteles arachnoides E. Geoffroy, 1806). American Journal of Primatology 23: 113-126.

- Strier KB (1991b). Demography and conservation of an endangered primate, Brachyteles arachnoides. Conservation Biology 5: 214-218.

Strier KB (1993/1994). Viability analyses of an isolated population of muriqui monkeys (Brachyteles arachnoides): implications for primate conservation and demography. Primate Conservation 14 15: $43-52$

- Strier KB (2000). Population viability and regional conservation priorities for muriquis (Brachyteles arachnoides) in Brazil's Atlantic Forest. Biotropica 32: 903-913.

- Strier KB (2005). Reproductive biology and conservation of muriquis. Neotropical Primates 13: 41-45.

- Strier KB, Ziegler TE (2000). Lack of pubertal influences on female dispersal in muriqui monkeys (Brachyteles arachnoides). Animal Behavior 59: 849-860.

- Strier KB, Boubli JP, Possamai CB, Mendes SL (2006). Population demography of northern muriquis (Brachyteles hypoxanthus) at the Estação Biológica de Caratinga/Reserva Particular do Patrimônio Natural Feliciano Miguel Abdala, Minas Gerais, Brazil. American Journal of Physical Anthropology 130: 227-237.

- Strier KB, Mendes SL, Santos RR (2001). Timing of births in sympatric brown howler monkeys (Alouatta fusca clamitans) and northern muriquis (Brachyteles arachnoides hypoxanthus). American Journal of Primatology 55: 87-100.

Tabacow FP, Mendes SL, Strier KB (2009). Spread of a terrestrial tradition in an arboreal primate. American Anthropologist 111: 238-249.

- Turner IM, Corlett RT (1996). The conservation value of small, isolated fragments of lowland tropical rain forest. Trends in Ecology and Evolution 11: 330-333.

Vié JC, Hilton-Taylor C, Stuart SN (2009). Wildlife in a Changing World: An Analysis of the 2008 IUCN Red List of Threatened Species. Gland, IUCN.

Waite TA, Vucetich T, Saurer T, Kroninger M, Vaughn E, Field K, Ibargüen S (2005). Minimizing extinction risk through genetic rescue. Animal Biodiversity and Conservation 28: 121-130. 